\title{
Aircraft Group Coordinated Flight Route Optimization Using Branch-and-Bound Procedure in Resolving the Problem of Environmental Monitoring
}

\author{
Georgy Lebedev ${ }^{1}$, Vladimir Goncharenko ${ }^{1}$, Denis Mikhaylin ${ }^{1}$, and Alena Rumakina ${ }^{1, *}$ \\ ${ }^{1}$ Moscow Aviation Institute (National Research University), 125080 Moscow, Russia
}

\begin{abstract}
The solution of a travelling salesman problem (TSP) for the case of two-dimensional flight routing is suggested. Unlike the case of one-dimensional routing, this solution implies the formation of two initial matrices of the distances between the points for two unlinked routes. The routes are analyzed alternately with the coordinated selection of the minimal length element in each of them. Examples of calculations demonstrating the proposed approach efficiency are provided indicating the reduction of overflights by more than two times.
\end{abstract}

\section{Introduction}

At the current time, more attention is paid to increasing efficiency of in-flight group action for various operation and control tasks. These include services for a given set of monitoring land points. The problem is solved by group of aircrafts $(A C)$, which routes should be predetermined based on the criteria of minimum total length.

Many works are devoted to the optimal onedimensional routing problem [1-3], among which the branch-and-bound algorithm plays a specific role [4] Group flight action penalties should be taken into consideration when crossing one-dimensional flight routes [5]. Otherwise, predictable flight control is required to ensure mutual safe approach. In this article the problem of routing of two AC coordinated flight is considered [6].

\section{Statement of the problem}

1. The problem of two horizontal flight open loop routes for two flying vehicles with common preset initial and finite points A and B, respectively, is solved. (Example in figure 1).

2. The set of fixed monitoring points is given. Coordinates of their location and total number (number $n=5$ in figure 1). It allows to form the parent matrix $M_{0}$ of distances between them, the beginning 0 and the end $(n+1)$ according to branch-and-bound procedure. In table 1 this matrix has the following form.

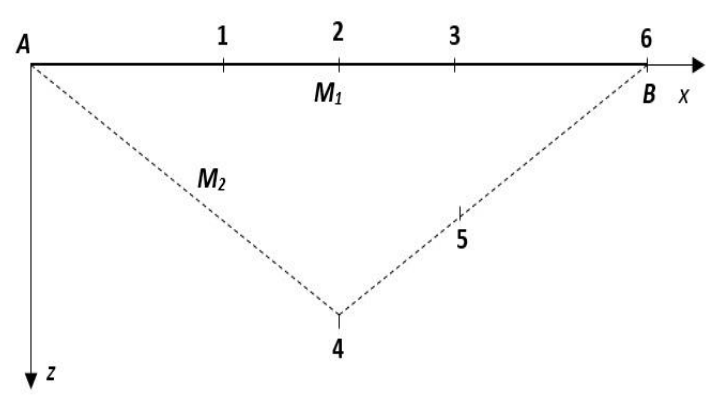

Fig. 1. Location of monitoring land points with the indication of calculated routes $M_{1}$ and $M_{2}$

Table 1. Initial matrix $M_{0}$.

\begin{tabular}{|c|c|c|c|c|c|c|c|}
\hline & 0 & 1 & 2 & 3 & 4 & 5 & 6 \\
\hline 0 & $X$ & 5 & 8 & 11 & 9,4 & 11,5 & $X$ \\
\hline 1 & $X$ & $X$ & 3 & 6 & 5,8 & 6,7 & 11 \\
\hline 2 & $X$ & 3 & $X$ & 3 & 5 & 4,3 & 8 \\
\hline 3 & $X$ & 6 & 3 & $X$ & 5,8 & 3 & 5 \\
\hline 4 & $X$ & 5,8 & 5 & 5,8 & $X$ & 3,5 & 9,4 \\
\hline 5 & $X$ & 6,7 & 4,3 & 3 & 3,5 & $X$ & 5,8 \\
\hline 6 & $X$ & $X$ & $X$ & $X$ & $X$ & $X$ & $X$ \\
\hline
\end{tabular}

This matrix contains forbidden elements as it is accepted for the well-known branch-and-bound [4], namely:

- matrix diagonal elements, forbidding any point penetration once more right after its servicing; 
- elements in the first column forbidding return to the initial point for released routes;

- matrix low line elements forbidding any further movement after final point $(n+1)$ penetration;

- the element $(0, n+1)$ forbidding the movement straightway form the initial point to the final.

1. Each point should be serviced once and by a single $A C$.

2. Formation of two flight paths so that their total length would be minimal is required.

\section{Numerical solution of two-dimensional routing problem by branch-and-bound modified procedure}

The solution of the problem is based on the well-known manipulations accepted for integer-valued branch-andbound procedure for one-dimensional routing. New necessary operations have been added. As in the wellknown procedure, the planning should consist of multiple steps of calculation $(k=1, \ldots, n+1)$. The difference is that instead of one matrix distance $M_{0}$, two matrices $M_{1}$ and $M_{2}$ are analyzed. One matrix per each route. The initial state of these matrices is equal to the state of the parent matrix (as shown below). However, attaching a new column to each of them to indicate coordinates $x$ and $z$ of the so-called "center of attraction" of both formed routes with numbers $(n+2)$ and $(n+3)$.

To explain the centers of attraction of the routes in the figure 2. the approximation of trajectories routes $M_{1}$ and $M_{2}$ is shown. It consists of two rectangular segments emanating from the origin and the end of the routes to the centers.

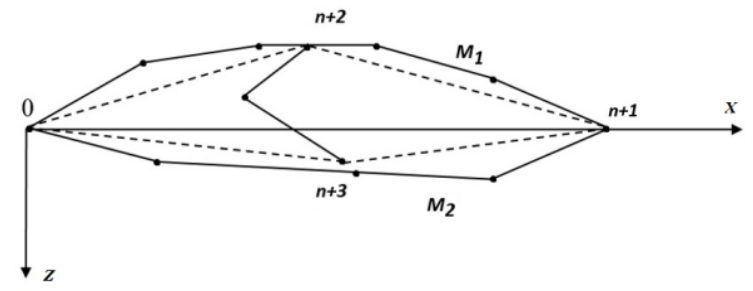

Fig. 2. Triangular approximation of the routes $M_{1}$ and $M_{2}$

We define this approximation as a triangular model. Its elements are dotted in rectangular segments.

At each computing step, we suggest to perform the following operations. Accentuating the new ones among them:

- alternately select one of the two matrices for the analysis. Find the element of the minimum length. This element should be included in the plan, as it is accepted for branch-and-bound;

- analyze the element obtained $(i, j)$ in "nearness" to the first or the second route;

- in the selected one of the two matrices delete as usual the $i$ row and the $j$ row. Insert forbidding character into $(j, i)$ element. After attachment of the element $(i$, $j$ ) you should additionally refine $z$ and $x$ coordinates of the corresponding route center of attraction. Insert them into the right column of the selected matrix.

- two rows $(i, j)$ and two columns $(i, j)$ should be deleted in the other matrix to exclude their servicing.

As a result, the dimension of both matrices can be reduced. After repetitions the number of updates of each matrix may be unequal. But the sum of these numbers at the end of calculation is equal to $(n+1)$. In the end, the proposed approach found answers to two questions:

- which of the two matrices have to be analyzed for the elements of short length;

- which of the two matrices should contain it.

According to the principle of the equality the elements of both matrices are analyzed alternately. But then the joining element belongs to the route to which it is "nearer," no matter what matrix was selected.

At the first stage of calculating the proximity measure is determined by the difference between distances $m 1$ and $m 2$ from element $(i, j)$ to the centers of attraction of $(n+2)(n+3)$ of the two routes, as shown in figure 2. The result of these calculations makes it possible to reduce the order of both matrices. This process would be repeated until both matrices move to a single element after $(n+1)$. In addition $z$ and $x$ coordinates of the centers of attraction would be separated from each other by the distance sufficient for the second stage of computations.

At the second stage of computations, additional criteria of proximity of each point to selected routes are implemented. They allow to form the joint list of twodimensional routing algorithm operations.

\section{Examples of the coordinated planning of the two routes close to each other}

The first example shows the effect of disposal of jumpers and the usage of triangular model of the routes trajectories (figure 3).

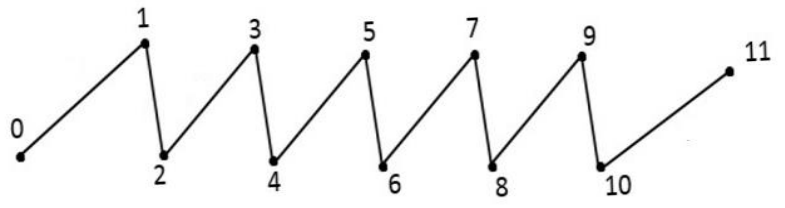

Fig. 3. An example of the trajectory in one-dimensional routing

Accordingly, a plan for a two-dimensional routing is shown in the figure 4. Flight time of one of the aircraft was reduced more than twice by eliminating "jumpers" between the two routes.

The figure 4 shows also the final allocation of the centers of attraction of the triangular flight trajectory approximation model (dashed lines № 12 and № 13).

The second example - environmental monitoring of 30 cities (a total length of 1,500 km) in the Moscow region using two aircraft. The task execution time was reduced by one and a half while during such flight. 


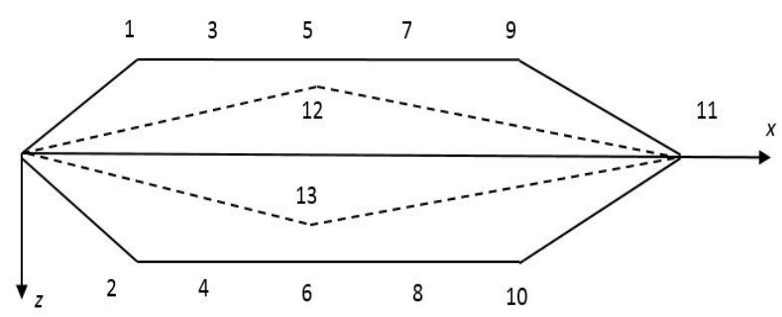

Fig. 4. The trajectories of two flight routes at the second calculation step

The two-stage calculation can be used for the task of coordinated selection of different flight route numbers $(N)$. In that case, $N$ matrices are replicated from the original matrix of distances. Then each of new matrices is to be_analyzed alternately.

At each step of calculation a single element with minimum length is selected $(i, j)$. It is assigned to one of the routes by the first criterion of proximity. Whereby new coordinates of its center of attraction are calculated, lines $i, j$ and columns $i, j$ in the other matrices are excluded. As a result, the trajectory centers of attraction will be uneven. It is sufficient to continue the solution of the problem.

\section{Conclusions}

As a tool for training the neural network, the method of solving the task of two-dimensional routing is proposed. This method is based on the initial matrix of distances between points of the few matrices for each open-loop route formation. The matrices are analyzed alternately with coordinated selection of the minimum length element in each of them.

It is shown that in comparison to one-dimensional routing, neural network two-dimensional route planning allows to reduce the overflight time in 1.5-2 times.

\section{References}

1. G.N. Lebedev etc., New technology. Mechatronics, automation, control, 11, 60-65 (2009)

2. G.N. Lebedev, A.V. Efimov, Proceedings of Samara state aerospace University, 1, 63-70 (2012)

3. G.N. Lebedev, L.A. Mirzoyan, New technologies. Mechatronics, automation, control, 12, 24-28 (2011)

4. L.T. Kuzin, Foundations of cybernetics (Moscow, Energy, 1973)

5. G.N. Lebedev, Zaw Min Taik. New technologies. Mechatronics, automation, control, 5, 61-68 (2014)

6. G.N. Lebedev, V.I. Goncharenko, A.V. Rumakina, New technologies. Mechatronics, automation, control, 17(11), 783-791 (2016) 